\title{
Latent profile analysis of dietary intake in a community-dwelling sample of older Americans
}

Nicholas J Bishop ${ }^{1, *}$, Krystle E Zuniga ${ }^{2}$ and Christina M Ramirez ${ }^{3}$

${ }^{1}$ Family and Child Development, School of Family and Consumer Sciences, Texas State University, 601 University Drive, San Marcos, TX 78666, USA: ${ }^{2}$ Department of Oncology, Dell Medical School, University of Texas at Austin, Austin, TX, USA: ${ }^{3}$ Nutrition and Foods, School of Family and Consumer Sciences, Texas State University, San Marcos, TX, USA

Submitted 20 September 2018: Final revision received 27 February 2019: Accepted 28 March 2019: First published online 28 June 2019

\begin{abstract}
Objective: To estimate latent dietary profiles in a community-dwelling sample of older Americans and identify associations between dietary profile membership and individual demographic, socio-economic and health characteristics.

Design: Secondary analysis of the 2012 Health and Retirement Study (HRS) and linked 2013 Health Care and Nutrition Study (HCNS). Latent profile analysis identified mutually exclusive subgroups of dietary intake and bivariate analyses examined associations between dietary profile membership, participant characteristics and nutrient intakes.

Setting: USA.

Participants: An analytic sample of 3558 adults aged 65 years or older.

Results: Four dietary profiles were identified with $15.5 \%$ of the sample having a 'Healthy' diet, 42.0\% consuming a 'Western' diet, 29.7\% having a diet consisting of high intake of all food groups and $12.7 \%$ reporting relatively low intake of all food groups. Members of the 'Healthy' profile reported the greatest socioeconomic resources and health, and members of the 'Low Intake' profile had the fewest resources and worst health outcomes. Macronutrient and micronutrient intakes varied across profile although inadequate and excessive intakes of selected nutrients were observed for all profiles.

Conclusions: We identified dietary patterns among older Americans typified by either selective intake of foods or overall quantity of foods consumed, with those described as 'Low Intake' reporting the fewest socio-economic resources, greatest risk of food insecurity and the worst health outcomes. Limitations including the presence of measurement error in dietary questionnaires are discussed. The causes and consequences of limited dietary intake among older Americans require further study and can be facilitated by the HRS and HCNS.
\end{abstract}

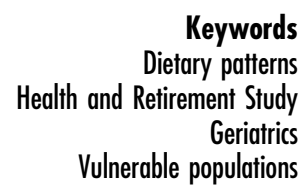

As the world's population of adults aged 60 years or older is projected to more than double between 2015 and $2050^{(1)}$, identifying modifiable risk factors of age-related chronic disease can help reduce the public health burden of population ageing. The association between diet quality and age-related chronic disease is well established ${ }^{(2-4)}$ with a growing focus on overall dietary intake as opposed to specific nutrients and foods, acknowledging potential interactions between nutrients and bioavailability of foods ${ }^{(5)}$. Classifications of overall diet quality are either defined a priori, consisting of indices used to score and rank diet quality, or are estimated a posteriori, using data-reduction techniques such as factor or cluster analysis ${ }^{(6)}$. Knowledge- based dietary classifications including the Mediterranean diet, the Healthy Eating Index and the Dietary Approaches to Stop Hypertension score have been associated with numerous chronic disease outcomes ${ }^{(7-12)}$, although predefined dietary indices have limitations including various definitions of a 'healthy' diet based on current nutritional guidance and the inability to describe heterogeneous dietary patterns that may exist among distinct population subgroups ${ }^{(6)}$.

Empirical approaches to describing dietary intake rely on statistical modelling to identify common dietary patterns in the population of interest and do not depend on pre-established dietary indices ${ }^{(6)}$. Dietary patterns identified through data-reduction techniques have been 
associated with a host of health behaviours and chronic diseases $^{(5,6,11,13)}$ although most existing studies are based on smaller, non-representative samples or come from studies that lack robust measurement of factors associated with diet including socio-economic status (SES), food insecurity, and health outcomes such as chronic disease and physical disability. Existing research that has applied empirical classification to diet among older adults has been conducted in countries such as Australia and Spain ${ }^{(14,15)}$ or draws from non-generalizable samples of older Americans ${ }^{(7,16)}$. Finally, the statistical methods commonly used to classify dietary patterns among older adults are typically limited to factor analytic methods that do not identify mutually exclusive groups that can be easily compared, or clustering approaches with strict assumptions that do not allow formal tests of model fit ${ }^{(17)}$.

As the understanding of current dietary patterns among ageing Americans is limited, the objective of our study was to identify common dietary patterns among older Americans and investigate associations between dietary habits, demographic and socio-economic characteristics, health behaviours, chronic disease, and intake of macronutrients and micronutrients known to be associated with chronic disease outcomes. We draw from recently collected dietary data linked to rich contextual data representative of America's community-dwelling older adults and use latent profile analysis, a form of finite mixture modelling, to overcome limitations common to a posteriori methods of dietary classification.

\section{Methods}

Observations were drawn from the Health and Retirement Study (HRS), a biennial national panel survey of older Americans beginning in 1992 and funded by the National Institute on Aging (NIA) and the Social Security Administration (grant number NIA U01AG009740) ${ }^{(18)}$. The 2013 Health Care and Nutrition Study (HCNS), an offyear mail-out HRS supplement conducted by the Survey Research Center at the Institute for Social Research at the University of Michigan (grant number NIA U01AG009740), collected information about food consumption using the Harvard FFQ originally proposed by Willett and colleagues ${ }^{(19,20)}$ with calculated energy and nutrient totals based on Harvard School of Public Health nutrient tables ${ }^{(21)}$. The University of Michigan's institutional review board approved the HRS protocol and participants were read a confidentiality statement and provided oral or implied consent when first contacted, and signed a written informed consent form at each interview ${ }^{(22)}$. All measures were collected through participant self-report and proxy response to the HRS and HCNS surveys was allowed when the respondent was unable to complete the interview. The HRS sample is a multistage, area-clustered and stratified sampling design representative of all age-eligible noninstitutionalized individuals in the US population ${ }^{(23)}$.

The original HCNS contained 8073 observations with complete information on consumption of 164 food items. Individuals under the age of 65 years at time of completing the HCNS ( $n$ 3792) were removed. To reduce the impact of measurement error on our estimates of interest, we excluded respondents who had daily energy intakes falling outside the commonly used allowable range of 2092-14 $644 \mathrm{~kJ} / \mathrm{d}(500-3500 \mathrm{kcal} / \mathrm{d})$ for women and 3347-16 736 $\mathrm{kJ} / \mathrm{d}(800-4000 \mathrm{kcal} / \mathrm{d})$ for men $(n 286)^{(24)}$, or who had either the 2012 HRS or 2013 HCNS survey completed by a proxy respondent $(n 437)$, resulting in an analytic sample of 3558. Participant characteristics were measured in 2012 and were primarily drawn from the RAND HRS data file $(\text { Version } \mathrm{P})^{(25)}$, with indicators of cognitive status taken from the core HRS files. Items used to identify food insecurity were drawn from the HCNS. The simple response rates for the HRS and HCNS were 89.1 and $65.0 \%$, respectively ${ }^{(20,26)}$.

\section{Measures}

Respondents were asked to indicate average total consumption of each specified food item and amount over the past 12 months. Measures of average consumption of 164 food items were converted to responses reflecting average servings per day. Food items were grouped and five food items were excluded from analysis based on recommendations provided by the Food Patterns Equivalence Database by the US Department of Agriculture ${ }^{(27)}$. The remaining 159 food items were grouped based on nutritional similarity, then summed to represent daily intake of thirty-five separate food groups. Table 1 describes the food groups and lists excluded food items. For clarity, we use the term 'food groups' to describe the thirty-five separate foods and food groups used to identify dietary profiles. Scores were log-transformed with an offset of 0.01 to improve normality and allow inclusion of individuals reporting non-intake of a given food group.

Demographic and socio-economic characteristics, health behaviours and health outcomes were compared across dietary profiles. Demographic measures included respondents' age, gender $(1=$ female, $0=$ male $)$, race/ ethnicity (White, Black, Hispanic, Other), marital status ( 1 = partnered or married, $0=$ single, divorced or widowed) and retirement status ( $1=$ retired, $0=$ not retired). Education ( $<12$ years of education, 12 years of education, $>12$ years of education), longest occupational tenure (white-collar, blue-collar, female homemaker, other occupational tenure), and log-transformed household income and assets were included as indicators of SES. Additionally, food insecurity was assessed using the US Household Food Security Survey Module six-item short form $^{(28)}$. Responses to the six items were summed then dichotomized to reflect either food security (raw score of 
Table 1 Food groupings used in the dietary profile analysis, 2012 Health and Retirement Study/2013 Health Care and Nutrition Study*

\begin{tabular}{|c|c|}
\hline Food groups & Food items \\
\hline Red meat & Lean hamburger, regular hamburger, beef/pork/lamb - mixed, pork main dish, beef/lamb main dish \\
\hline Processed meats & Bacon, beef/pork hot dogs, chicken/turkey hot dogs, processed meat, processed meat other \\
\hline French fries & French fries \\
\hline Refined grains & White bread, bagels, muffins/biscuits, rice white, pasta \\
\hline Sweets & Milk chocolate, dark chocolate, candy bars, candy without chocolate, reduced-fat cookies \\
\hline Potatoes & Potatoes \\
\hline Pizza & Pizza \\
\hline Snacks & Potato chips, crackers, crackers wholegrain, crackers other, popcorn light \\
\hline Butter and margarine & Butter, spreadable butter, margarine \\
\hline Other vegetables & Onions raw, onions cooked, corn, mixed vegetables, summer squash \\
\hline Dark yellow vegetables & Carrots raw, carrots cooked, winter squash, yams/sweet potatoes \\
\hline Leafy vegetables & Spinach cooked, spinach raw, head lettuce, leaf lettuce \\
\hline Cruciferous vegetables & Broccoli, cabbage, cauliflower, Brussels sprouts, kale/mustard/chard greens \\
\hline Fruit & Raisins or grapes, prunes/dried plums, apple sauce, apples/pears, apricots \\
\hline Tomatoes & Tomatoes, tomato or v8 juice, tomato sauce, salsa \\
\hline Legumes & Beans or lentils, tofu soya protein, peas or lima beans \\
\hline Alcohol & Beer regular, beer light, red wine, white wine, liquor \\
\hline Coffee & Coffee with caffeine, decaffeinated coffee, dairy coffee drink \\
\hline Cold cereal & Cold cereal \\
\hline Condiments & $\begin{array}{l}\text { Non-dairy cream, jams/preserves/honey, ketchup/red chilli sauce, salt added, number of teaspoons } \\
\text { of sugar }\end{array}$ \\
\hline Cream soup & Cream soup \\
\hline Eggs & Eggs regular, egg whites, eggs fortified \\
\hline Fish and seafood & Tuna canned, fish sticks, seafood main dish, fish dark, fish other \\
\hline Fruit juice & Prune juice, apple juice, orange juice fortified, orange juice regular, grapefruit juice \\
\hline High-fat dairy & Whole milk, cream, regular ice cream, cottage/ricotta cheese, cream cheese \\
\hline High-energy drinks & Carbonated with caffeine and sugar, carbonated with sugar other, sugar beverage other \\
\hline Low-fat dairy & Skimmed milk, 1 or $2 \%$ milk, soya milk, frozen yoghurt/low-fat ice cream, flavoured yoghurt \\
\hline Low-energy drinks & Low-calorie carbonated with caffeine, low-calorie carbonated without caffeine \\
\hline $\begin{array}{l}\text { Mayonnaise and creamy } \\
\text { dressing }\end{array}$ & Mayonnaise regular, salad dressing \\
\hline Nuts & Peanut butter, peanuts, walnuts, nuts other \\
\hline Olive oil & Olive oil \\
\hline Organ meat & Liver beef/pork, liver chicken/turkey \\
\hline Poultry & Chicken/turkey, chicken/turkey with skin, chicken/turkey without skin \\
\hline Tea & Tea with caffeine, decaffeinated tea \\
\hline Whole grains & Wholegrain bread, rice brown, oatmeal, rye bread, cooked cereal other \\
\hline
\end{tabular}

${ }^{*}$ Food items excluded from analysis: Splenda, artificial sweetener, garlic, low-carbohydrate bars, plain water.

†Some individual food items were not collapsed into groups due to the combination of multiple food items used to produce the food (i.e. pizza) or having varying preparation techniques (i.e. potatoes).

0-1) or low/very low food security (raw score of 2-6; $1=$ food insecure, $0=$ food secure).

Health behaviours included BMI (underweight $\left(\mathrm{BMI}<18.5 \mathrm{~kg} / \mathrm{m}^{2}\right)$, normal weight $\left(18.5 \mathrm{~kg} / \mathrm{m}^{2} \leq \mathrm{BMI}\right.$ $\left.<25 \mathrm{~kg} / \mathrm{m}^{2}\right)$, overweight $\left(25 \mathrm{~kg} / \mathrm{m}^{2} \leq \mathrm{BMI}<30 \mathrm{~kg} / \mathrm{m}^{2}\right)$, obese $\left(\mathrm{BMI} \geq 30 \mathrm{~kg} / \mathrm{m}^{2}\right)$ ), vigorous physical activity (participation in activities such as sports and heavy housework, or a job that involves physical labour; no vigorous physical activity; vigorous physical activity less than 1 time/week; vigorous physical activity more than 1 time/week), current smoking status ( $1=$ current smoker, $0=$ not current smoker) and alcohol consumption (non-drinkers; moderate drinkers (men drinking between 1 and 14 drinks/week, women drinking between 1 and 7 drinks/week); heavy drinkers (men drinking more than 14 drinks/week, women drinking more than 7 drinks/(week)).

Health status was measured using self-rated health status ( $1=$ poor, $5=$ excellent $)$ and a sum of doctor-diagnosed chronic conditions (high blood pressure, cancer, diabetes, lung disease, heart problems, stroke, psychiatric problems, arthritis). Early physical disability was assessed with a sum of eleven indicators of limitation in physical mobility (any difficulty in each of the following activities: stooping or crouching, climbing one flight of stairs without resting, climbing several flights of stairs without resting, moving large objects, sitting in a chair for two hours, getting up from a chair after sitting for long periods, lifting weights of more than $4.5 \mathrm{~kg}(10 \mathrm{lb})$, raising arms above shoulder level, walking one block, walking several blocks, picking up a dime from a table). Advanced physical disability was based on report of any limitation in activities of daily living (ADL; some difficulty in bathing activities, getting dressed, eating, getting in and out of bed, or walking across a room; $1=$ any ADL limitation, $0=$ no ADL limitation). Cognitive status was assessed with a multidimensional cognitive measure based on the Telephone Interview for Cognitive Status (TICS) and included tests of immediate and delayed word recall, a serial 7 counting task, backwards counting task, date naming, word recognition and general knowledge questions (range $0-35)^{(29,30)}$.

Energy intake, macronutrient density, macronutrient intake and micronutrient intake were compared across 
Table 2 Model fit statistics for latent profile analysis by number of estimated profile, 2012 Health and Retirement Study/2013 Health Care and Nutrition Study*

\begin{tabular}{|c|c|c|c|c|c|c|c|c|c|}
\hline \multirow[b]{2}{*}{ Latent profile } & \multirow[b]{2}{*}{ df } & \multicolumn{5}{|c|}{ Model fit statistic } & \multicolumn{3}{|c|}{ VLMR } \\
\hline & & LL & $\mathrm{AIC}$ & $\mathrm{BIC}$ & SS-BIC & Entropy & Mean & SD & $P$ \\
\hline 1 & 70 & -196671 & 393481 & 393913 & 393691 & & - & - & - \\
\hline 2 & 106 & -192703 & 385617 & 386272 & 385935 & 0.82 & $448 \cdot 3$ & $407 \cdot 9$ & $<0.001$ \\
\hline 3 & 142 & -190714 & 381711 & 382589 & 382138 & $0 \cdot 84$ & $549 \cdot 7$ & $617 \cdot 0$ & $<0.001$ \\
\hline 4 & 178 & -189696 & 379748 & 380848 & 380282 & 0.83 & 435.5 & 901.8 & 0.038 \\
\hline 5 & 214 & -189038 & 378506 & 379827 & 379147 & 0.82 & $1079 \cdot 4$ & $1265 \cdot 2$ & 0.426 \\
\hline
\end{tabular}

LL, log-likelihood; AIC, Akaike information criterion, BIC, Bayesian information criterion; SS-BIC, sample-size-adjusted Bayesian information criterion; VLMR, Vuong-LoMendell-Rubin likelihood ratio test.

${ }^{*}$ All estimates and statistical tests account for the complex survey design.

dietary patterns. Estimated daily energy intake was measured as average daily total kilocalorie intake. Macronutrient density for daily intake of carbohydrate, protein, fat and saturated fat was calculated as the estimated percentage of daily energy coming from each macronutrient source. Macronutrients examined included fibre, $n-3$ fatty acids, EPA, DHA, alcohol, added sugar, n-6 fatty acids, trans fat and cholesterol. Micronutrients included $\mathrm{Ca}$, $\mathrm{Fe}, \mathrm{K}$, folate and vitamins $\mathrm{B}_{12}, \mathrm{C}, \mathrm{D}$ and $\mathrm{E}$. To assess dietary diversity, we included a count of the number of different food groups each participant reported consuming. Finally, RMR was estimated using the Mifflin-St Jeor ${ }^{(31)}$ equation, identified as the most accurate equation to estimate RMR in older populations ${ }^{(32)}$. RMR was also used to calculate the ratio of daily energy intake to RMR.

\section{Statistical methods}

Latent profile analysis (LPA) was used to classify older adults into mutually exclusive dietary patterns across the thirty-five observed food groups. LPA is a form of the more general latent class analysis (also described as finite mixture modelling), allowing identification of unobserved heterogeneity in multiple continuous response variables $^{(33)}$. The best-fitting latent profile model was determined using information criteria-based metrics including the Akaike information criterion, Bayesian information criterion and sample-size-adjusted Bayesian information criterion, and the Vuong, Lo, Mendell and Rubin likelihood ratio test allowed a significance test of whether the inclusion of an additional profile contributed to a significantly better-fitting model $(P<0.05$ was used as the $\alpha$ level for nested model-fit testing). To ensure convergence on global maxima through several replications of the best log-likelihood for each model, 10000 random sets of starting values with ten final-stage optimizations were used. When random starting values and final-stage optimizations were doubled, log-likelihoods were replicated for all reported LPA models. For reporting purposes, exploratory factor analysis was used to identify meaningfully correlated food groups. Although exploratory factor analysis with orthogonal rotation identified nine factors with eigenvalues $>1$, only two factors had three or more items with loadings
$>0 \cdot 40$. Mplus version $8.1^{(34)}$ was used to conduct the LPA and exploratory factor analysis using maximum-likelihood estimation with robust SE and adjustments for missing data and complex survey design.

Once the optimal number of latent dietary profiles was identified, respondent characteristics and indicators of energy, macronutrient density and macronutrient and micronutrient intakes were compared across dietary profile using the statistical software package SAS version $9.4^{(35)}$. Overall differences in continuous measures were compared across dietary profile using ANOVA with bivariate follow-up through least-squares mean differences. Differences in categorical measures across dietary profile were tested using contingency tables with the Rao-Scott $\chi^{2}$ test. Significant overall $\chi^{2}$ tests were partitioned into $2 \times 2$ contingency tables with RaO-Scott $\chi^{2}$ tests used to test statistical significance and OR were used to identify the direction the observed cell frequency departed from the expected cell frequency. To reduce the likelihood of type I error due to multiple comparisons, the significance level for each follow-up test was adjusted using the Bonferroni correction. All statistical tests adjusted for complex survey design.

\section{Results}

\section{Latent profile analysis}

Table 2 presents model fit statistics for LPA models estimating between one and five latent profiles. As the number of estimated profiles increased, the Akaike information criterion, Bayesian information criterion and samplesize-adjusted Bayesian information criterion generally decreased, while entropy remained consistently above 0.80. The Vuong, Lo, Mendell and Rubin likelihood ratio test indicated that the four-class model fit the data significantly better than the three-class solution $(P=0.038)$, but the five-class solution did not significantly improve model fit over the four-class solution $(P=0 \cdot 426)$. Based on model fit tests and the goal of parsimony, the four-class solution was identified as the best description of latent dietary profiles. 
Table 3 Average servings per day of food items by latent dietary profile for American adults aged 65 years or older, 2012 Health and Retirement Study/2013 Health Care and Nutrition Study*

\begin{tabular}{|c|c|c|c|c|c|c|c|c|c|c|}
\hline & \multicolumn{8}{|c|}{ Latent dietary profile } & \multirow[b]{3}{*}{$\mathrm{F \dagger}$} & \multirow[b]{3}{*}{$P \dagger$} \\
\hline & \multicolumn{2}{|c|}{ 'Healthy' } & \multicolumn{2}{|c|}{ 'Western' } & \multicolumn{2}{|c|}{ 'High Intake' } & \multicolumn{2}{|c|}{ 'Low Intake' } & & \\
\hline & Mean & sD & Mean & sD† & Mean & sD† & Mean & sD† & & \\
\hline \multicolumn{11}{|l|}{ Vegetables, fruits and legumes } \\
\hline Other vegetables & $1 \cdot 3^{a}$ & 0.9 & $0.7^{b}$ & 0.4 & $1 \cdot 6^{\mathrm{c}}$ & 0.8 & $0.3^{d}$ & 0.3 & $1015 \cdot 4$ & $<0.001$ \\
\hline Dark yellow vegetables & $0.5^{\mathrm{a}}$ & 0.5 & $0.2^{b}$ & 0.2 & $0.5^{\mathrm{a}}$ & 0.4 & $0.1^{\mathrm{c}}$ & 0.1 & 681.9 & $<0.001$ \\
\hline Leafy vegetables & $0.7^{a}$ & 0.5 & $0.3^{\mathrm{b}}$ & 0.3 & $0.7^{\mathrm{c}}$ & 0.5 & $0.1^{d}$ & 0.1 & 739.5 & $<0.001$ \\
\hline Cruciferous vegetables & $0.5^{\mathrm{a}}$ & 0.4 & $0.2^{\mathrm{b}}$ & 0.2 & $0.5^{\mathrm{a}}$ & 0.4 & $0.1^{\mathrm{c}}$ & 0.1 & $600 \cdot 2$ & $<0.001$ \\
\hline Fruit & $2 \cdot 1^{a}$ & 1.3 & $1.2^{\mathrm{b}}$ & 0.9 & $2 \cdot 1^{a}$ & 1.3 & $0.8^{\mathrm{c}}$ & 0.8 & 374.0 & $<0.001$ \\
\hline Tomatoes & $0.6^{\mathrm{a}}$ & 0.5 & $0.4^{\mathrm{b}}$ & 0.4 & $0.8^{\mathrm{C}}$ & 0.5 & $0.2^{d}$ & 0.2 & $376 \cdot 4$ & $<0.001$ \\
\hline Legumes & $0.3^{a}$ & 0.3 & $0 \cdot 1^{\mathrm{b}}$ & 0.1 & $0.3^{\mathrm{c}}$ & 0.3 & $0.1^{d}$ & 0.1 & $354 \cdot 2$ & $<0.001$ \\
\hline \multicolumn{11}{|l|}{ Red meat and processed foods } \\
\hline Red meat & $0.2^{\mathrm{a}}$ & 0.2 & $0.4^{\mathrm{b}}$ & 0.3 & $0.6^{c}$ & 0.4 & $0.3^{d}$ & 0.2 & $439 \cdot 4$ & $<0.001$ \\
\hline Processed meat & $0.1^{a}$ & 0.2 & $0.4^{\mathrm{b}}$ & 0.3 & $0.5^{c}$ & 0.4 & $0.2^{d}$ & 0.4 & $445 \cdot 1$ & $<0.001$ \\
\hline French fries & $0.0^{\mathrm{a}}$ & 0.0 & $0.1^{\mathrm{b}}$ & 0.1 & $0.1^{\mathrm{b}}$ & 0.1 & $0.1^{\mathrm{c}}$ & 0.1 & $195 \cdot 8$ & $<0.001$ \\
\hline Refined grains & $0.5^{\mathrm{a}}$ & 0.5 & $1 \cdot 1^{\mathrm{b}}$ & 1.0 & $1 \cdot 2^{\mathrm{c}}$ & 0.9 & $0.9^{d}$ & 0.9 & $184 \cdot 8$ & $<0.001$ \\
\hline Sweets & $0.7^{a}$ & 0.7 & $1.4^{\mathrm{b}}$ & 1.5 & $1.7^{\mathrm{c}}$ & 1.5 & $1.0^{\mathrm{d}}$ & 1.2 & $201 \cdot 7$ & $<0.001$ \\
\hline Potatoes & $0.1^{a}$ & 0.2 & $0.2^{\mathrm{b}}$ & 0.2 & $0.3^{c}$ & 0.2 & $0.1^{a}$ & 0.1 & $243 \cdot 1$ & $<0.001$ \\
\hline Pizza & $0.0^{\mathrm{a}}$ & 0.0 & $0.1^{b}$ & 0.1 & $0.1^{\mathrm{c}}$ & 0.1 & $0.0^{\mathrm{d}}$ & 0.0 & $189 \cdot 8$ & $<0.001$ \\
\hline Snacks & $0.4^{\mathrm{a}}$ & 0.4 & $0.6^{\mathrm{b}}$ & 0.6 & $0.8^{\mathrm{C}}$ & 0.7 & $0.4^{\mathrm{a}}$ & 0.5 & $215 \cdot 0$ & $<0.001$ \\
\hline Butter/margarine & $0.5^{a}$ & 0.7 & $1 \cdot 1^{\mathrm{b}}$ & $1 \cdot 2$ & $1.3^{\mathrm{c}}$ & $1 \cdot 2$ & $0.7^{d}$ & 1.0 & $217 \cdot 6$ & $<0.001$ \\
\hline \multicolumn{11}{|l|}{ Ungrouped foods } \\
\hline Alcohol & $0.4^{\mathrm{a}}$ & 0.7 & $0.4^{\mathrm{a}}$ & 0.9 & $0.5^{\mathrm{b}}$ & 0.9 & $0.3^{c}$ & 0.7 & $35 \cdot 8$ & $<0.001$ \\
\hline Coffee & $1 \cdot 3^{\mathrm{a}}$ & 1.4 & $1.6^{\mathrm{b}}$ & 1.4 & $1.8^{\mathrm{b}}$ & 1.4 & $1 \cdot 2^{\mathrm{a}}$ & $1 \cdot 3$ & $34 \cdot 7$ & $<0.001$ \\
\hline Cold cereal & $0.3^{a}$ & 0.4 & $0.3^{\mathrm{b}}$ & 0.4 & $0.3^{\mathrm{b}}$ & 0.4 & $0.3^{a}$ & 0.5 & $27 \cdot 2$ & $<0.001$ \\
\hline Condiments & $1 \cdot 2^{\mathrm{a}}$ & 1.3 & $1.9^{b}$ & 1.8 & $2 \cdot 1^{c}$ & 1.8 & $1.6^{\mathrm{d}}$ & 1.5 & 77.7 & $<0.001$ \\
\hline Cream soup & $0.0^{a}$ & 0.0 & $0.0^{\mathrm{b}}$ & 0.1 & $0.1^{\mathrm{c}}$ & 0.1 & $0.0^{\mathrm{a}}$ & 0.0 & 157.9 & $<0.001$ \\
\hline Eggs & $0.4^{\mathrm{a}}$ & 0.4 & $0.4^{\mathrm{a}}$ & 0.5 & $0.6^{\mathrm{b}}$ & 0.5 & $0.4^{\mathrm{c}}$ & 0.5 & 117.5 & $<0.001$ \\
\hline Fish seafood & $0.3^{a}$ & 0.3 & $0.2^{b}$ & 0.1 & $0.3^{\mathrm{c}}$ & 0.2 & $0.1^{d}$ & 0.1 & 221.6 & $<0.001$ \\
\hline Fruit juice & $0.4^{a}$ & 0.7 & $0.5^{\mathrm{b}}$ & 0.6 & $0.7^{\mathrm{c}}$ & 0.7 & $0.4^{\mathrm{a}}$ & 0.6 & 89.7 & $<0.001$ \\
\hline High-fat dairy & $0.6^{\mathrm{a}}$ & 0.6 & $1.0^{\mathrm{b}}$ & 0.9 & $1 \cdot 2^{\mathrm{c}}$ & 0.9 & $0.6^{\mathrm{a}}$ & 0.7 & $219 \cdot 9$ & $<0.001$ \\
\hline High-energy drinks & $0.1^{a}$ & 0.2 & $0.4^{\mathrm{b}, \mathrm{c}}$ & 0.7 & $0.3^{b, d}$ & 0.5 & $0.4^{\mathrm{c}, \mathrm{d}}$ & 0.7 & $127 \cdot 3$ & $<0.001$ \\
\hline Low-fat dairy & $1.0^{\mathrm{a}}$ & 1.0 & $0.8^{\mathrm{b}}$ & 0.9 & $1.0^{\mathrm{a}}$ & 1.0 & $0.8^{\mathrm{c}}$ & 1.0 & 47.9 & $<0.001$ \\
\hline Low-energy drinks & $0.1^{a}$ & 0.4 & $0 \cdot 3^{b, c}$ & 0.6 & $0.3^{\mathrm{b}}$ & 0.6 & $0.3^{c}$ & 0.7 & $52 \cdot 8$ & $<0.001$ \\
\hline Mayonnaise and cream dressing & $0.4^{a}$ & 0.5 & $0.4^{b}$ & 0.4 & $0.7^{\mathrm{c}}$ & 0.6 & $0.2^{\mathrm{d}}$ & 0.4 & $148 \cdot 1$ & $<0.001$ \\
\hline Nuts & $0.8^{\mathrm{a}}$ & 0.9 & $0.5^{\mathrm{b}}$ & 0.6 & $0.9^{c}$ & 0.8 & $0.4^{\mathrm{d}}$ & 0.7 & 158.4 & $<0.001$ \\
\hline Olive oil & $0.3^{a}$ & 0.7 & $0.1^{\mathrm{b}}$ & 0.2 & $0 \cdot 2^{\mathrm{a}, \mathrm{c}}$ & 0.6 & $0.1^{\mathrm{c}}$ & 0.5 & $184 \cdot 7$ & $<0.001$ \\
\hline Organ meat & $0.0^{\mathrm{a}}$ & 0.1 & $0.0^{\mathrm{b}}$ & $0 \cdot 1$ & $0 \cdot 0^{\mathrm{b}}$ & $0 \cdot 1$ & $0.0^{\mathrm{a}}$ & 0.1 & $25 \cdot 1$ & $<0.001$ \\
\hline Poultry & $0.3^{\mathrm{a}}$ & 0.3 & $0.3^{\mathrm{b}}$ & 0.3 & $0.4^{\mathrm{c}}$ & 0.3 & $0.2^{\mathrm{a}}$ & 0.2 & $172 \cdot 7$ & $<0.001$ \\
\hline Tea & $0.8^{a}$ & 1.2 & $0.4^{\mathrm{b}}$ & 0.9 & $0.6^{\mathrm{a}}$ & 1.1 & $0.3^{c}$ & 0.9 & 94.4 & $<0.001$ \\
\hline Whole grains & $1 \cdot 2^{\mathrm{a}}$ & $1 \cdot 1$ & $0.7^{b}$ & 0.8 & $1 \cdot 2^{\mathrm{C}}$ & $1 \cdot 0$ & $0.6^{d}$ & 0.9 & $159 \cdot 5$ & $<0.001$ \\
\hline Analytic sample size & \multirow{2}{*}{\multicolumn{2}{|c|}{$\begin{array}{c}552 \\
5436762\end{array}$}} & \multirow{2}{*}{\multicolumn{2}{|c|}{$\begin{array}{c}1495 \\
14740699\end{array}$}} & \multirow{2}{*}{\multicolumn{2}{|c|}{$\begin{array}{c}1058 \\
10170789\end{array}$}} & \multirow{2}{*}{\multicolumn{2}{|c|}{$\begin{array}{c}453 \\
4686946\end{array}$}} & & \\
\hline Estimated population size & & & & & & & & & & \\
\hline
\end{tabular}

a,b,c,d Mean values within row with unlike superscript letters were significantly different ( $\alpha$ level for statistical significance identified with Bonferroni correction). ${ }^{*}$ All estimates and statistical tests excluding SD account for the complex survey design.

†Mean and SD reported for non-log-transformed food groups, statistical tests based on log-transformed food groups.

Average servings per day of the thirty-five food groups estimated separately for each latent profile are presented in Table 3. Two factors were identified through exploratory factor analysis which we describe as 'vegetables, fruits and legumes', containing seven food groups, and 'red meat and processed foods', containing nine food groups. Nineteen food items were not meaningfully associated with intake of other foods and are ordered alphabetically. The four latent dietary profiles were characterized by either the types of foods being consumed or the amounts of foods being consumed. Of the estimated 35035196 US adults aged 65 years or over in 2013 based on the HCNS population adjustments for our analytic sample, 15.5\% ( $n 552$;
N5 436 762) were classified as eating a 'Healthy' diet, $42.1 \%$ ( $n 1495 ; N 14740699)$ were classified as consuming a 'Western' diet, 29.0 \% ( $n$ 1058; $N 10170789)$ were classified as having 'High Intake' and $13.4 \%$ ( $n$ 453; $N 4686946)$ were described as having 'Low Intake'. The US Census Bureau reports an estimated population of US adults aged 65 years or older in 2013 as 44672695 , indicating that our analyses underestimate the US resident population of older adults by about $22 \%{ }^{(36)}$.

Older adults identified as having a 'Healthy' diet had relatively high intake of vegetables, fruits and legumes, low intake of red meat and processed foods, low intake of condiments and energy drinks, and high intake of low-fat dairy, 
Table 4 Respondent characteristics by latent dietary profile for American adults aged 65 years or older, 2012 Health and Retirement Study/ 2013 Health Care and Nutrition Study ${ }^{\star}$

\begin{tabular}{|c|c|c|c|c|c|c|c|c|c|c|}
\hline & \multicolumn{8}{|c|}{ Latent dietary profile } & \multirow[b]{3}{*}{$\mathrm{F} \dagger$} & \multirow[b]{3}{*}{$P \dagger$} \\
\hline & \multicolumn{2}{|c|}{ 'Healthy' } & \multicolumn{2}{|c|}{ 'Western' } & \multicolumn{2}{|c|}{ 'High Intake' } & \multicolumn{2}{|c|}{ 'Low Intake' } & & \\
\hline & Mean & sD $†$ & Mean & sD & Mean & sD† & Mean & sD $†$ & & \\
\hline \multicolumn{11}{|l|}{ Continuous measures } \\
\hline Age & $75 \cdot 2^{a, b, c}$ & $6 \cdot 9$ & $74 \cdot 6^{a, d}$ & $7 \cdot 2$ & $75 \cdot 1^{\mathrm{b}, \mathrm{d}, \mathrm{e}}$ & $6 \cdot 9$ & $75 \cdot 9^{\mathrm{c}, \mathrm{e}}$ & 7.5 & 3.5 & 0.016 \\
\hline Household income $\ddagger$ & $67 \cdot 5^{a}$ & $97 \cdot 8$ & $57 \cdot 7^{a}$ & 69.8 & $71 \cdot 3^{\mathrm{b}}$ & $113 \cdot 2$ & $46 \cdot 5^{\mathrm{b}}$ & $66 \cdot 8$ & $24 \cdot 0$ & $<0.001$ \\
\hline Household assets $\ddagger$ & $691 \cdot 6^{\mathrm{a}, \mathrm{b}}$ & 1054.9 & $445 \cdot 1^{\mathrm{a}}$ & $946 \cdot 4$ & $616 \cdot 2^{\mathrm{b}}$ & $1055 \cdot 6$ & $379 \cdot 0^{c}$ & $850 \cdot 3$ & 13.8 & $<0.001$ \\
\hline Self-rated health & $3 \cdot 4^{\mathrm{a}}$ & 1.0 & $3 \cdot 2^{\mathrm{b}}$ & 1.0 & $3 \cdot 3^{\mathrm{c}}$ & 0.9 & $3.0^{\mathrm{d}}$ & 1.1 & $18 \cdot 8$ & $<0.001$ \\
\hline Chronic conditions & $2 \cdot 3^{a}$ & 1.3 & $2 \cdot 5^{b, c}$ & 1.4 & $2 \cdot 5^{\mathrm{b}, \mathrm{d}}$ & 1.4 & $2 \cdot 6^{\mathrm{c}, \mathrm{d}}$ & 1.4 & 5.4 & .001 \\
\hline Mobility limitations & $2 \cdot 2^{\mathrm{a}}$ & 2.5 & $3 \cdot 0^{\mathrm{b}}$ & 2.9 & $2 \cdot 8^{\mathrm{c}}$ & $2 \cdot 8$ & $3 \cdot 4^{d}$ & $3 \cdot 3$ & $14 \cdot 3$ & $<0.001$ \\
\hline \multirow[t]{2}{*}{ TICS score§ } & $15 \cdot 6^{a}$ & $4 \cdot 4$ & $14 \cdot 9^{b}$ & $4 \cdot 0$ & $15 \cdot 4^{b}$ & $4 \cdot 1$ & $13 \cdot 6^{c}$ & 4.5 & 23.7 & $<0.001$ \\
\hline & \multicolumn{2}{|c|}{$\%$} & \multicolumn{2}{|c|}{$\%$} & \multicolumn{2}{|c|}{$\%$} & \multicolumn{2}{|c|}{$\%$} & $x^{2}$ & $P$ \\
\hline \multicolumn{11}{|l|}{ Categorical measures } \\
\hline Female & \multirow{2}{*}{\multicolumn{2}{|c|}{$73.1 \|$}} & \multirow{2}{*}{\multicolumn{2}{|c|}{53.81}} & \multirow{2}{*}{\multicolumn{2}{|c|}{$55 \cdot 8$}} & \multirow{2}{*}{\multicolumn{2}{|c|}{61.5}} & $50 \cdot 8$ & $<0.001$ \\
\hline Race/ethnicity & & & & & & & & & & \\
\hline White & \multicolumn{2}{|c|}{$79.4 \prod$} & \multicolumn{2}{|c|}{85.7} & \multicolumn{2}{|c|}{$89.6 \|$} & & & 43.8 & $<0.001$ \\
\hline Black & & & & & & & & & & \\
\hline Hispanic & & & & & & & & & & \\
\hline Other & & & & & & & & & & \\
\hline Married/partnered & & & & & & & & & $42 \cdot 1$ & $<0.001$ \\
\hline Retired & & & & & & & & & $9 \cdot 2$ & 0.027 \\
\hline Education & & & & & & & & & & \\
\hline$<$ HS degree & & & & & & & & & $70 \cdot 9$ & $<0.001$ \\
\hline HS degree & & & & & & & & & & \\
\hline$>\mathrm{HS}$ degree & & & & & & & & & & \\
\hline Occupational tenure & & & & & & & & & & \\
\hline White-collar & & & & & & & & & 63.8 & $<0.001$ \\
\hline Blue-collar & & & & & & & & & & \\
\hline Homemaker & & & & & & & & & & \\
\hline Other & & & & & & & & & & \\
\hline $\begin{array}{l}\text { Food insecurity } \\
\text { BMI }\end{array}$ & & & & & & & & & 41.5 & $<0.001$ \\
\hline Underweight & & & & & & & & & 41.6 & 0.001 \\
\hline Normal & & & & & & & & & & \\
\hline Overweight & & & & & & & & & & \\
\hline Obese & & & & & & & & & & \\
\hline Current smoker & & & & & & & & & 34.6 & $<0.001$ \\
\hline Alcohol consumption & & & & & & & & & & \\
\hline None & & & & & & & & & $32 \cdot 3$ & $<0.001$ \\
\hline Moderate & & & & & & & & & & \\
\hline Heavy & & & & & & & & & & \\
\hline Vigorous activity & & & & & & & & & & \\
\hline None & & & & & & & & & $90 \cdot 2$ & $<0.001$ \\
\hline Some & & & & & & & & & & \\
\hline Regular & & & & & & & & & & \\
\hline Any ADL limitation & & & & & & & & & $23 \cdot 5$ & $<0.001$ \\
\hline
\end{tabular}

TICS, Telephone Interview of Cognitive Status; HS, high school; ADL, activities of daily living.

$\mathrm{a}, \mathrm{b}, \mathrm{c}, \mathrm{d}, \mathrm{e}$ Mean values within row with unlike superscript letters were significantly different ( $\alpha$ level for statistical significance identified with Bonferroni correction).

${ }^{*}$ All estimates and statistical tests excluding SD account for the complex survey design.

†Mean and SD reported for non-log-transformed food groups, statistical tests based on log-transformed food groups.

¥Mean and SD reported for non-log-transformed household income and assets, statistical tests based on log-transformed household income and assets. Mean and SD for household income and assets are reported in thousands of \$US.

§Overall sample size for TICS was 3394 owing to some items used in the summary score not being asked of all Health and Retirement Study participants.

|l Observed cell frequency significantly greater than expected.

१Observed cell frequency significantly less than expected.

amount of vegetables, fruits and legumes, red meat and processed foods, and nearly all other foods. Finally, those identified in the 'Low Intake' profile had the lowest intake of vegetables, fruits and legumes of all identified profiles, relatively low intake of red meat and processed foods, and generally the lowest reported intake of all other food condiments, high-fat dairy and high-energy drinks. Older adults classified as 'High Intake' consumed the greatest nuts, olive oil, tea and whole grains. Those with a 'Western' diet were typified by low intake of vegetables, fruits and legumes, relatively high intake of red meat and processed foods, as well as comparatively high intake of coffee, 
Table 5 Energy intake, macronutrient density, macronutrient intake and micronutrient intake by latent dietary profile for American adults aged 65 years or older, 2012 Health and Retirement Study/2013 Health Care and Nutrition Study*

\begin{tabular}{|c|c|c|c|c|c|c|c|c|c|c|}
\hline & \multicolumn{8}{|c|}{ Latent dietary profile } & & \\
\hline & \multicolumn{2}{|c|}{ 'Healthy' } & \multicolumn{2}{|c|}{ 'Western' } & \multicolumn{2}{|c|}{ 'High Intake' } & \multicolumn{2}{|c|}{ 'Low Intake' } & \multicolumn{2}{|c|}{ ANOVA } \\
\hline & Mean & SD & Mean & SD & Mean & SD & Mean & SD & $F$ & $P$ \\
\hline Total energy $(\mathrm{kJ} / \mathrm{d})$ & $6239 \cdot 2^{\mathrm{a}}$ & $2156 \cdot 9$ & $6778 \cdot 1^{\mathrm{b}}$ & $2096 \cdot 2$ & $9546 \cdot 2^{c}$ & $2351 \cdot 8$ & $4817.9^{d}$ & $1906 \cdot 6$ & 677.8 & $<0.001$ \\
\hline Total energy (kcal/d) & $1491 \cdot 2^{\mathrm{a}}$ & $515 \cdot 5$ & $1620 \cdot 0^{b}$ & $501 \cdot 0$ & $2281 \cdot 6^{c}$ & $562 \cdot 1$ & $1151 \cdot 5^{d}$ & $455 \cdot 7$ & $677 \cdot 8$ & $<0.001$ \\
\hline \multicolumn{11}{|l|}{ Macronutrient density } \\
\hline Carbohydrates (\%E) & $54 \cdot 6^{\mathrm{a}}$ & $9 \cdot 1$ & $52 \cdot 5^{\mathrm{b}}$ & $8 \cdot 1$ & $51 \cdot 4^{\mathrm{c}}$ & $6 \cdot 7$ & $54.9^{a}$ & 9.9 & 29.5 & $<0.001$ \\
\hline Protein (\%E) & $17 \cdot 2^{\mathrm{a}}$ & 3.7 & $16 \cdot 3^{\mathrm{b}}$ & 3.3 & $16 \cdot 9^{\mathrm{a}, \mathrm{b}}$ & $2 \cdot 8$ & $16 \cdot 0^{c}$ & 3.8 & $18 \cdot 4$ & $<0.001$ \\
\hline Fat $(\% \mathrm{E})$ & $33 \cdot 3^{a}$ & $7 \cdot 6$ & $35 \cdot 0^{\mathrm{b}}$ & $6 \cdot 1$ & $36 \cdot 0^{c}$ & $5 \cdot 3$ & $33 \cdot 0^{\mathrm{a}}$ & $7 \cdot 7$ & 35.5 & $<0.001$ \\
\hline Saturated fat (\%E) & $10 \cdot 3^{a}$ & $2 \cdot 6$ & $12 \cdot 2^{b}$ & $2 \cdot 7$ & $12 \cdot 1^{\mathrm{c}}$ & $2 \cdot 2$ & $11.4^{\mathrm{b}}$ & 3.1 & $87 \cdot 1$ & $<0.001$ \\
\hline \multicolumn{11}{|l|}{ Macronutrient intake } \\
\hline Fibre $(\mathrm{g})$ & $22 \cdot 1^{\mathrm{a}}$ & $9 \cdot 6$ & $15 \cdot 6^{\mathrm{b}}$ & $5 \cdot 2$ & $25 \cdot 6^{c}$ & $7 \cdot 7$ & $10 \cdot 3^{d}$ & 4.8 & 773.1 & $<0.001$ \\
\hline$n$-3 Fatty acids (g) & $1.5^{\mathrm{a}}$ & 1.0 & $1.3^{\mathrm{b}}$ & $0 . \overline{6}$ & $2 \cdot 0^{c}$ & 0.9 & $0.9^{d}$ & 0.6 & $295 \cdot 3$ & $<0.001$ \\
\hline $\operatorname{EPA}(\mathrm{mg})$ & $44 \cdot 9^{a}$ & 54.5 & $24 \cdot 3^{b}$ & 23.7 & $43 \cdot 9^{a}$ & $42 \cdot 3$ & $13 \cdot 2^{c}$ & $21 \cdot 4$ & $128 \cdot 1$ & $<0.001$ \\
\hline $\mathrm{DHA}(\mathrm{mg})$ & $129 \cdot 5^{a}$ & $128 \cdot 3$ & $78 \cdot 6^{\mathrm{b}}$ & $62 \cdot 6$ & $133 \cdot 7^{a}$ & $100 \cdot 3$ & $48 \cdot 7^{\mathrm{c}}$ & 57.4 & $161 \cdot 1$ & $<0.001$ \\
\hline Alcohol (g) & $5 \cdot 2^{a, b, c}$ & $9 \cdot 7$ & $5 \cdot 6^{\mathrm{a}, \mathrm{d}}$ & $12 \cdot 0$ & $7 \cdot 5^{\mathrm{b}, \mathrm{d}}$ & $12 \cdot 6$ & $4 \cdot 0^{\mathrm{C}}$ & $10 \cdot 0$ & $10 \cdot 0$ & $<0.001$ \\
\hline Added sugar (g) & $30 \cdot 2^{\mathrm{a}}$ & $20 \cdot 7$ & $50 \cdot 3^{b}$ & 34.4 & $58 \cdot 1^{\mathrm{c}}$ & $31 \cdot 4$ & $40 \cdot 6^{d}$ & 34.4 & 105.5 & $<0.001$ \\
\hline$n-6$ Fatty acids $(\mathrm{g})$ & $10 \cdot 3^{a}$ & $5 \cdot 8$ & $10 \cdot 3^{a}$ & 4.4 & $15 \cdot 2^{b}$ & $5 \cdot 8$ & $7 \cdot 1^{\mathrm{c}}$ & 4.3 & 339.0 & $<0.001$ \\
\hline Trans fat $(\mathrm{g})$ & $1.0^{\mathrm{a}}$ & 0.4 & $1.7^{\mathrm{b}}$ & 0.8 & $2 \cdot 2^{\mathrm{c}}$ & 0.9 & $1 \cdot 2^{d}$ & 0.7 & 387.5 & $<0.001$ \\
\hline Cholesterol (g) & $170 \cdot 2^{a}$ & 94.4 & $225 \cdot 5^{\mathrm{b}}$ & 114.0 & $307 \cdot 1^{c}$ & $117 \cdot 6$ & $156 \cdot 1^{a}$ & $108 \cdot 4$ & 281.4 & $<0.001$ \\
\hline \multicolumn{11}{|l|}{ Micronutrient intake } \\
\hline $\mathrm{Ca}(\mathrm{mg})$ & $707 \cdot 3^{a}$ & $502 \cdot 4$ & $683.5^{\mathrm{b}}$ & 485.0 & $923.5^{c}$ & 455.8 & $548 \cdot 4^{\mathrm{a}}$ & $452 \cdot 8$ & $95 \cdot 7$ & $<0.001$ \\
\hline $\mathrm{Fe}(\mathrm{mg})$ & $12 \cdot 3^{a}$ & 5.6 & $12 \cdot 6^{\mathrm{b}}$ & 5.2 & $17 \cdot 3^{c}$ & 5.6 & $9 \cdot 2^{\mathrm{a}}$ & $5 \cdot 1$ & 319.2 & $<0.001$ \\
\hline $\mathrm{K}(\mathrm{mg})$ & $2874 \cdot 6^{a}$ & $1061 \cdot 8$ & $2455 \cdot 0^{\mathrm{b}}$ & 730.4 & $3681 \cdot 1^{\mathrm{C}}$ & 923.3 & $1723.9^{d}$ & $655 \cdot 7$ & 744.8 & $<0.001$ \\
\hline Folate $(\mu \mathrm{g})$ & $7 \cdot 5^{a}$ & 3.3 & $5 \cdot 4^{b}$ & 1.8 & $8.7^{\mathrm{c}}$ & $2 \cdot 7$ & $3.5^{\mathrm{d}}$ & 1.6 & 766.4 & $<0.001$ \\
\hline Vitamin $\mathrm{B}_{12}(\mu \mathrm{g})$ & $0 \cdot 1^{a}$ & 0.1 & $0 \cdot 1^{\mathrm{b}}$ & $0 \cdot 1$ & $0.2^{\mathrm{c}}$ & $0 \cdot 1$ & $0.1^{d}$ & 0.1 & $159 \cdot 3$ & $<0.001$ \\
\hline Vitamin C (mg) & $115 \cdot 8^{a}$ & $69 \cdot 6$ & $89 \cdot 2^{b}$ & 55.4 & $140 \cdot 8^{c}$ & 65.5 & $58 \cdot 1^{d}$ & 54.4 & $260 \cdot 1$ & $<0.001$ \\
\hline Vitamin D $(\mu \mathrm{g})$ & $4 \cdot 3^{\mathrm{a}}$ & 3.6 & $3 \cdot 7^{b}$ & 2.5 & $4.9^{\mathrm{c}}$ & 2.9 & $3 \cdot 2^{d}$ & 2.9 & 55.4 & $<0.001$ \\
\hline Vitamin E (mg) & $7 \cdot 6^{a}$ & $4 \cdot 3$ & $6 \cdot 0^{\mathrm{b}}$ & $2 \cdot 6$ & $9 \cdot 5^{\mathrm{c}}$ & 3.7 & $4 \cdot 1^{d}$ & $2 \cdot 6$ & 408.4 & $<0.001$ \\
\hline \multicolumn{11}{|l|}{ Diet diversity } \\
\hline Number of foods & $29 \cdot 6^{a}$ & $2 \cdot 8$ & $31 \cdot 5^{b}$ & $2 \cdot 0$ & $32 \cdot 5^{c}$ & 1.7 & $29 \cdot 0^{d}$ & 3.0 & 361.6 & $<0.001$ \\
\hline \multicolumn{11}{|l|}{ RMR } \\
\hline Estimated RMR (kJ/d) & $5310 \cdot 8^{a}$ & $1064 \cdot 4$ & $5904 \cdot 9^{b}$ & $1199 \cdot 6$ & $5869 \cdot 5^{c}$ & 1193.4 & $5670 \cdot 6^{\mathrm{b}}$ & $1142 \cdot 2$ & $36 \cdot 6$ & $<0.001$ \\
\hline Estimated RMR (kcal/d) & $1269 \cdot 3^{a}$ & 254.4 & $1411 \cdot 3^{b}$ & $286 \cdot 7$ & $1409 \cdot 3^{c}$ & $285 \cdot 2$ & $1355 \cdot 3^{b}$ & 273.0 & $36 \cdot 6$ & $<0.001$ \\
\hline Daily energy intake:RMR & $1 \cdot 2^{\mathrm{a}}$ & 0.5 & $1 \cdot 2^{\mathrm{a}}$ & 0.5 & $1 \cdot 7^{\mathrm{b}}$ & 0.5 & $0.9^{c}$ & 0.4 & 381.9 & $<0.001$ \\
\hline Analytic sample size & \multicolumn{2}{|c|}{552} & \multicolumn{2}{|c|}{1495} & \multicolumn{2}{|c|}{1058} & \multicolumn{2}{|c|}{453} & & \\
\hline Estimated population size & \multicolumn{2}{|c|}{5436762} & \multicolumn{2}{|c|}{14740699} & \multicolumn{2}{|c|}{10170789} & \multicolumn{2}{|c|}{4686946} & & \\
\hline
\end{tabular}

$\% \mathrm{E}$, percentage of energy.

$\mathrm{a}, \mathrm{b}, \mathrm{c}, \mathrm{d}$ Mean values within row with unlike superscript letters were significantly different ( $\alpha$ level for statistical significance identified with Bonferroni correction).

*All estimates and statistical tests excluding SD account for the complex survey design.

groups excluding non-nutrient-dense items such as condiments and energy drinks.

\section{Respondent characteristics by dietary profile}

Table 4 describes how demographic characteristics, SES, health behaviours and health indicators were associated with dietary profile membership. Age did not appear to differ meaningfully across dietary profile. Members of the 'Healthy' dietary pattern were more likely to be female, less likely to be White, and less likely to be married/partnered and retired than expected. Household income and assets were relatively high among 'Healthy' dietary pattern members, as was the likelihood of earning greater than a highschool education and reporting white-collar occupational tenure. Older adults in the 'Healthy' dietary profile were likely not smokers, tended towards normal BMI, reported regular vigorous activity more often than those in other profiles, and generally had the best physical and cognitive health of all profiles. The 'Western' dietary profile had significantly fewer women than expected by chance and had household income and assets similar to those in the 'Healthy' profile. Members of the 'Western' profile were less likely to report earning greater than a high-school degree than expected and had a relatively high likelihood of reporting blue-collar occupational tenure. There were relatively high proportions of smokers and respondents reporting no vigorous physical activity in the 'Western' profile, and members of this group appeared to have on average worse health than members of the 'Healthy' profile but health similar to members of the 'High Intake' profile.

Older adults in the 'High Intake' dietary profile had the greatest likelihood of being White, being married or partnered, and had the greatest reported household income 
of all profiles, but otherwise had SES similar to those in the 'Healthy' profile. Those in the 'High Intake' profile were relatively unlikely to report currently smoking, were the most likely to report moderate alcohol consumption, and had a relatively high likelihood of reporting vigorous physical activity. Members of the 'High Intake' profile had lower self-rated health and TICS cognitive scores, more chronic health conditions, and greater likelihood of reporting an ADL limitation than those in the 'Healthy' profile. Finally, the 'Low Intake' dietary profile had a greater proportion of Hispanic and Other race/ethnicity respondents than expected, and members had a relatively low likelihood of being married. 'Low Intake' older adults also reported the fewest monetary resources, were the most likely to report not having a high-school degree, and the least likely to report white-collar occupational tenure of all dietary profiles. The 'Low Intake' profile was also characterized by the greatest likelihood of food insecurity, smoking and reporting no physical activity. 'Low Intake' older adults also generally had the worst health status observed, with $20.0 \%$ of respondents reporting at least one limitation in ADL.

\section{Energy, macronutrient and micronutrient intakes by dietary profile}

Table 5 presents estimated daily energy intake, macronutrient density, macronutrient intake, micronutrient intake, and both RMR and the ratio of estimated daily energy intake to RMR by dietary profile. Energy intake differed significantly across all profiles with the 'High Intake' group having an average energy intake $2768.1 \mathrm{~kJ}$ (661.6 kcal) greater than next highest group, the 'Western' profile. Carbohydrate and protein macronutrient density were generally similar across dietary profile and members of the 'Western' and 'High Intake' profiles had the greatest saturated fat energy density. Older adults in the 'High Intake' profile had the greatest consumption of fibre, overall $n-3$ fatty acids, added sugar, $n-6$ fatty acids, trans fat and cholesterol. Compared with the 'High Intake' group, 'Healthy' profile members consumed significantly less $n$ - 6 fatty acids, trans fat and cholesterol. Individuals with a 'Western' diet had relatively high intake of added sugar, trans fat and cholesterol considering that their average energy intake was similar to members of the 'Healthy' profile. The 'Low Intake' profile had on average the lowest intake of macronutrients although members consumed more added sugar than those in the 'Healthy' profile. Members of the 'High Intake' profile had the greatest micronutrient intake while members of the 'Low Intake' profile generally had the lowest intake of micronutrients. Across all dietary patterns, intakes of $\mathrm{Ca}$, K, folate, vitamin $\mathrm{D}$ and vitamin $\mathrm{E}$ were below Dietary Reference Intakes. Members of the 'Western' and 'High Intake' profiles reported consuming a similar number of foods, with those in the 'Low Intake' profile having the least diverse food intake. Estimated RMR was lower in the 'Healthy' profile than in other profiles, and regarding the energy intake to RMR ratio, members of the 'High Intake' profile had estimated energy intake about $70 \%$ greater than RMR. The energy intake to RMR ratio for members of the 'Healthy' and 'Western' profiles was about $1.2(\mathrm{SD} 0.5)$, and that for members of the 'Low Intake' group was $0.9(\operatorname{SD} 0 \cdot 4)$.

\section{Discussion}

Using a sample of community-dwelling US adults aged 65 years or older, we identified four latent dietary profiles that were characterized by either type or quantity of foods being consumed. Profile membership was associated with the respondents' demographic and socio-economic characteristics, health behaviours and indicators of health status. Macronutrient and micronutrient intakes varied as expected across dietary profile, although intake of several key nutrients fell below acceptable ranges for all groups. Our findings support the use of empirical approaches to identify dietary patterns in diverse populations and call for further analyses of the association between empirically derived dietary patterns, socio-economic context and health outcomes among older adults using the HRS and HCNS.

The latent dietary patterns we identified in the HCNS reflect two distinct taxonomies of dietary intake. The first is based on preferential selection of certain food groups over others. Members of the 'Healthy' dietary profile appeared to selectively consume foods known to support health and well-being, while members of the 'Western' dietary profile displayed low intake of vegetables and fruits and high intakes of red meat and processed foods. SES and health behaviours were associated with dietary pattern membership, with 'Healthy' profile members reporting relatively high SES and favourable health behaviours compared with 'Western' profile members. The 'Western' dietary pattern contained the greatest proportion of respondents, a concerning outcome given established associations between 'Western' dietary habits and increased risk of chronic disease ${ }^{(37-39)}$.

The remaining dietary profiles were characterized by the overall amount of food being consumed. The 'High Intake' dietary profile contained the second-largest proportion of older adults and was characterized by the highest daily consumption of almost all food groups. The 'High Intake' group had relatively high SES and reported generally healthful behaviours, but also reported lower subjective health, greater chronic disease burden, lower cognitive scores, and had a greater likelihood of reporting an ADL limitation than those in the 'Healthy' profile.

The final and potentially most problematic dietary pattern identified was characterized by overall low food intake, likely reflecting a population at risk of nutritional deficiencies and poor health. 'Low Intake' older adults consumed the fewest vegetables, fruits and legumes of all profiles, reported relatively low consumption of red 
meat and processed foods, and intake of other foods was also generally low. The dietary habits and health behaviours of those in the 'Low Intake' profile occurred in a setting that reflected restricted access to socio-economic resources, which were coupled with the worst self-rated health, highest levels of physical disability and lowest cognitive scores of all dietary profiles. Notably, the average rate of food insecurity in this profile was $18.6 \%$, substantially greater than estimates drawn from comparably aged US populations ${ }^{(40,41)}$. Under-reporting of dietary intake may contribute to the low intake of foods observed in this group, although we believe older adults in the 'Low Intake' dietary profile appear particularly vulnerable and likely represent those who would most benefit from nutritional interventions.

Dietary profile membership was associated with differing macronutrient and micronutrient intakes that may place individuals at varying risk of chronic disease. The 'Western' and 'High Intake' patterns had the highest intakes of saturated fat, trans fat and cholesterol, likely related to high intake of red meat and processed foods. High intake of trans fat has been associated with increased risk of $\mathrm{CVD}^{(42,43)}$, and although the relationship between SFA and CVD is inconsistent, research suggests replacing SFA with PUFA may reduce the risk of $\mathrm{CVD}^{(42,43)}$. Fibre is associated with prevention or treatment of chronic diseases such as CVD, diabetes, colon cancer and obesity through health benefits that include weight control, regulation of blood glucose and lowering of cholesterol ${ }^{(44)}$. Daily fibre intake was below the Dietary Reference Intake in the 'Western' and 'Low Intake' patterns, reflecting insufficient consumption of whole grains, fruits and vegetables ${ }^{(45)}$.

Although the consumption of nutrients varied across profile, important similarities reflecting inadequate intakes of key nutrients emerged. All dietary patterns identified fell within the Acceptable Macronutrient Distribution Ranges for protein and carbohydrate ${ }^{(43)}$, although micronutrient adequacy is dependent on the nutrient density of the foods consumed. All dietary patterns were below $\mathrm{Ca}(1200 \mathrm{mg} / \mathrm{d})$ and vitamin D (15-20 $\mu \mathrm{g} / \mathrm{d}(600-800 \mathrm{IU} / \mathrm{d}))$ recommendations for older adults ${ }^{(45,46)}$. The risk for osteoporotic fractures increases with age and inadequate amounts of either Ca or vitamin D can increase fracture risk ${ }^{(47)}$. In addition, vitamin $\mathrm{D}$ deficiency has been shown to increase the risk for cognitive decline and dementia in older adults ${ }^{(48,49)}$. All profiles had low intakes of dairy products and few other foods contain Ca or vitamin D; therefore, adequate intakes of these nutrients may be difficult to achieve in older adults. Like other national estimates ${ }^{(50)}$, all dietary profiles had inadequate $\mathrm{K}$ intakes.

\section{Strengths and limitations}

The strengths of our study include the empirical identification of dietary profiles in a large sample of communitydwelling older Americans and analysis of an under-utilized data source allowing the linkage of dietary information with diverse indicators of SES and health. Compared with predefined dietary scores such as the Mediterranean diet or Healthy Eating Index, we identified dietary patterns reflecting both nutritional quality and overall quantity; outcomes that may be conflated when using predetermined dietary indices. Particularly important was the identification of 'Low Intake' older adults who appear to be at the greatest risk of nutritional deficits, low SES and poor health.

Our study also has important limitations to consider. The use of an FFQ to estimate dietary intake relies on the respondent to accurately report dietary intake, which could introduce measurement error through incorrect recall or different interpretations of the FFQ. Excluding proxy responses to the HRS and HCNS as well as removing observations with estimated daily energy intake falling outside the commonly used acceptable range have helped reduce the possible influence of measurement error on our findings, although our results may still be subject to bias due to under/over-reporting of dietary intake. In this context, the use of LPA to identify heterogeneous mixtures of dietary intake in the HCNS is an effective means of separating dietary responses distinguished by preferential consumption of certain types of foods (i.e. members of the 'Healthy' and 'Western' profiles) from those reporting either low or high intake of all foods. Those identified as members of dietary patterns distinguished by low or high intake should be further examined to differentiate those providing biased reports of dietary intake from those truly at risk of diseases related to excessive dietary intake or malnutrition. In particular, a combination of education, race/ ethnicity, and lower cognitive and physical function appear to be salient in identifying members of the 'Low Intake' group. Members of this group may have had different interpretations of the FFQ, difficulties in accurately completing the questionnaire, or potentially found the foods included in the FFQ less representative of their dietary intake.

In addition to the issue of measurement error, longitudinal measures of dietary intake would allow clearer identification of associations between dietary patterns, socio-economic context and health, and the ability to examine co-occurring changes in dietary habits and health over time would provide useful information about the efficacy of nutritional interventions. Also, being representative of only community-dwelling older Americans, the HRS and HCNS do not reflect the experiences of institutionalized older adults who are likely to be at the greatest risk of nutritional deficiencies and poor health. Exclusion of proxy responses likely magnifies the fact that our results are reflective of healthier, less-disabled older Americans. Additional studies are required to provide support for the representativeness of the dietary profiles we identified.

Our identification of dietary patterns among members of the rapidly expanding population of ageing Americans is a useful contribution for those who study the convergence of dietary habits, socio-economic constraints and health 
outcomes among older adults. The LPA approach to identifying heterogeneous dietary patterns proved useful in our research and should be applied to other sources of dietary data representing the diverse settings where older adults around the world live. Finally, the ability to link dietary information from the HCNS to longitudinal measures of SES and health in the HRS provides a unique opportunity to investigate the role of dietary intake in the changing health of America's ageing population.

\section{Acknowledgements}

Financial support: This research was supported by the Texas State University Research Enhancement Program. The Texas State University Research Enhancement Program had no role in the design, analysis or writing of this article. Conflict of interest: There are no conflicts of interest. Authorship: N.J.B. and K.E.Z. designed the research and N.J.B., K.E.Z. and C.M.R. wrote the paper; N.J.B. analysed the data and N.J.B. has primary responsibility for final content. All authors read and approved the final manuscript. Ethics of buman subject participation: This study was conducted according to the guidelines laid down in the Declaration of Helsinki and all procedures involving human subjects were approved by the University of Michigan's institutional review board. Verbal informed consent was obtained from all subjects and was witnessed and formally recorded.

Author ORCID. (D) Nicholas J Bishop, 0000-00021253-892X.

\section{References}

1. United Nations, Department of Economic and Social Affairs, Population Division (2015) World Population Ageing 2015. New York: UN.

2. Prince MJ, Wu F, Guo Y et al. (2015) The burden of disease in older people and implications for health policy and practice. Lancet 385, 549-562.

3. World Health Organization (2002) Diet, Nutrition and the Prevention of Chronic Diseases. Report of a Joint WHO/ FAO Expert Consultation. WHO Technical Report Series no. 916. Geneva: WHO.

4. Everitt AV, Hilmer SN, Brand-Miller JC et al. (2006) Dietary approaches that delay age-related diseases. Clin Interv Aging 1, 11-31.

5. Anderson AL, Harris TB, Tylavsky FA et al. (2011) Dietary patterns and survival of older adults. J Am Diet Assoc 111, 84-91.

6. Newby PK \& Tucker KL (2004) Empirically derived eating patterns using factor or cluster analysis: a review. Nutr Rev 62, 177-203.

7. Reedy J, Wirfalt E, Flood A et al. (2010) Comparing 3 dietary pattern methods - cluster analysis, factor analysis, and index analysis - with colorectal cancer risk. Am J Epidemiol 171, 479-487.
8. Benetou V, Trichopoulou A, Orfanos P et al. (2008) Conformity to traditional Mediterranean diet and cancer incidence: the Greek EPIC cohort. Br J Cancer 99, 191-195.

9. Bosire C, Stampfer MJ, Subar AF et al. (2013) Index-based dietary patterns and the risk of prostate cancer in the NIHAARP Diet and Health Study. Am J Epidemiol 177, 504-513.

10. Schwingshackl L \& Hoffmann G (2015) Diet quality as assessed by the Healthy Eating Index, the Alternate Healthy Eating Index, the Dietary Approaches to Stop Hypertension score, and health outcomes: a systematic review and meta-analysis of cohort studies. J Acad Nutr Diet 115, 780-800.

11. Van de Rest O, Berendsen AA, Haveman-Nies A et al. (2015) Dietary patterns, cognitive decline, and dementia: a systematic review. Adv Nutr 6, 154-168.

12. McCullough ML, Feskanich D, Stampfer MJ et al. (2002) Diet quality and major chronic disease risk in men and women: moving toward improved dietary guidance. Am J Clin Nutr 76, 1261-1271.

13. Kerver JM, Yang EJ, Bianchi L et al. (2003) Dietary patterns associated with risk factors for cardiovascular disease in healthy US adults. Am J Clin Nutr 78, 1103-1110.

14. Thorpe MG, Milte CM, Crawford D et al. (2016) A comparison of the dietary patterns derived by principal component analysis and cluster analysis in older Australians. Int J Behav Nutr Phys Act 13, 30.

15. Machón M, Mateo-Abad M, Vrotsou K et al. (2018) Dietary patterns and their relationship with frailty in functionally independent older adults. Nutrients 10, E406.

16. Wirfält E, Midthune D, Reed J et al. (2009) Associations between food patterns defined by cluster analysis and colorectal cancer incidence in the NIH-AARP Diet and Health Study. Eur J Clin Nutr 63, 707-717.

17. Sotres-Alvarez D, Herring AH \& Siega-Riz AM (2010) Latent class analysis is useful to classify pregnant women into dietary patterns. J Nutr 140, 2253-2259.

18. Hauser RM \& Weir D (2010) Recent developments in longitudinal studies of aging in the United States. Demography $\mathbf{4 7}$, Suppl., S111-S131.

19. Willett WC, Sampson L, Stampfer MJ et al. (1985) Reproducibility and validity of a semiquantitative food frequency questionnaire. Am J Epidemiol 122, 51-65.

20. Institute for Social Research, University of Michigan (2014) 2013 Health Care and Nutrition Study V5.0: nutrient totals data description. http://hrsonline.isr.umich.edu/modules/ meta/2013/hcns/desc/2013HCNS_data_description_nt.pdf (accessed September 2018).

21. Harvard T.H. Chan School of Public Health Nutrition (2015) Nutrient tables. http://regepi.bwh.harvard.edu/ health/ (accessed September 2018).

22. Weir DR, Langa KM, Ofstedal MB et al. (2017) Health and Retirement Study institutional review board information. http://hrsonline.isr.umich.edu/sitedocs/irb/HRS_IRB_ Information.pdf (accessed September 2018).

23. Ofstedal MB, Weir D, Chen K-T et al. (2011) HRS documentation report: updates to HRS sample weights. https://hrs.isr. umich.edu/sites/default/files/biblio/dr-013.pdf (accessed August 2018).

24. Willett WC (2013) Nutritional Epidemiology, 3rd ed. New York: Oxford University Press.

25. Chien S, Campbell N, Chan C et al. (2016) RAND HRS data documentation, version P. http://hrsonline.isr.umich.edu/ modules/meta/rand/randhrsp/randhrs_P.pdf (accessed September 2018).

26. Institute for Social Research, University of Michigan (2017) Health and Retirement Study: sample sizes and response rates. https://hrs.isr.umich.edu/sites/default/files/biblio/Response Rates_2017.pdf (accessed September 2018).

27. Bowman SA, Clemens JC, Friday JE et al. Food Patterns Equivalents Database 2013-14: methodology and user 
guide. http://www.ars.usda.gov/nea/bhnrc/fsrg (accessed September 2018).

28. US Department of Agriculture, Economic Research Service (2012) US Household Food Security Survey Module: six-item short form. https://www.ers.usda.gov/media/8282/ short2012.pdf (accessed September 2018).

29. Brandt J, Spencer M \& Folstein MF (1988) The Telephone Interview for Cognitive Status. Neuropsychiatry Neuropsychol Behav Neurol 1, 111-117.

30. Institute for Social Research, University of Michigan (2005) HRS/AHEAD documentation report. Documentation of cognitive functioning measures in the Health and Retirement Study. http://hrsonline.isr.umich.edu/sitedocs/userg/dr-006. pdf (accessed September 2018).

31. Mifflin MD, St Jeor ST, Hill LA et al. (1990) A new predictive equation for resting energy expenditure in healthy individuals. Am J Clin Nutr 51, 241-247.

32. Reidlinger DP, Willis JM \& Whelan K (2015) Resting metabolic rate and anthropometry in older people: a comparison of measured and calculated values. J Hum Nutr Diet 28, 72-84.

33. Collins LM \& Lanza ST (2010) Latent Class and Latent Transition Analysis: With Applications in the Social, Behavioral, and Health Sciences. Hoboken, NJ: John Wiley \& Sons.

34. Muthén LK \& Muthén BO (2017) Mplus User's Guide, 8th ed. Los Angeles, CA: Muthén \& Muthén.

35. SAS Institute Inc. (2015) SAS/STAT ${ }^{\circledR} 14.1$ User's Guide. Cary, NC: SAS Institute Inc.

36. Population Division, US Census Bureau (2018) Annual estimates of the resident population by single year of age and sex for the United States: April 1, 2010 to July 1, 2017. https://factfinder.census.gov/bkmk/table/1.0/en/PEP/2017/ PEPSYASEXN (accessed September 2018).

37. Zhang XY, Shu L, Si CJ et al. (2015) Dietary patterns, alcohol consumption and risk of coronary heart disease in adults: a meta-analysis. Nutrients 7, 6582-6605.

38. Leon-Munoz LM, Garcia-Esquinas E, Lopez-Garcia E et al. (2015) Major dietary patterns and risk of frailty in older adults: a prospective cohort study. BMC Med 13, 11.

39. Gougeon L, Payette H, Morais J et al. (2015) Dietary patterns and incidence of depression in a cohort of community-dwelling older Canadians. J Nutr Health Aging 19, 431-436.

40. Goldberg SL \& Mawn BE (2015) Predictors of food insecurity among older adults in the United States. Public Health Nurs 32, 397-407.

41. Strickhouser S, Wright JD \& Donley AM (2015) Food insecurity among older adults: a report submitted to AARP foundation. http://www.aarp.org/content/dam/aarp/aarp_foundation/ 2015-PDFs/AF-Food-Insecurity-2015Update-Final-Report. pdf (accessed September 2018).

42. Li Y, Hruby A, Bernstein AM et al. (2015) Saturated fats compared with unsaturated fats and sources of carbohydrates in relation to risk of coronary heart disease: a prospective cohort study. J Am Coll Cardiol 66, 1538-1548.

43. US Department of Agriculture \& US Department of Health and Human Services (2015) 2015-2020 Dietary Guidelines for Americans, 8th ed. https://health.gov/dietaryguidelines/ 2015/resources/2015-2020_Dietary_Guidelines.pdf (accessed September 2018).

44. Timm D \& Slavin JL (2008) Dietary fiber and the relationship to chronic diseases. Am J Lifestyle Med 2, 233-240.

45. Institute of Medicine (2005) Dietary Reference Intakes for Energy, Carbohydrate, Fiber, Fat, Fatty Acids, Cholesterol, Protein, and Amino Acids. Washington, DC: National Academy Press.

46. Institute of Medicine (2011) Dietary Reference Intakes for Calcium and Vitamin D. Washington, DC: National Academy Press.

47. Weaver CM, Alexander DD, Boushey CJ et al. (2016) Calcium plus vitamin D supplementation and risk of fractures: an updated meta-analysis from the National Osteoporosis Foundation. Osteoporos Int 27, 367-376.

48. Miller JW, Harvey DJ, Beckett LA et al. (2015) Vitamin D status and rates of cognitive decline in a multiethnic cohort of older adults. JAMA Neurol 72, 1295-1303.

49. Annweiler C, Dursun E, Féron F et al. (2015) Vitamin D and cognition in older adults: updated international recommendations. J Intern Med 277, 45-57.

50. Zhang Z, Cogswell ME, Gillespie C et al. (2013) Association between usual sodium and potassium intake and blood pressure and hypertension among US adults: NHANES 20052010. PLoS One 8, e75289. 